\title{
COUNTEREXAMPLE TO A CONJECTURE \\ ON THE ALGEBRAIC LIMIT CYCLES OF POLYNOMIAL VECTOR FIELDS
}

\author{
JAUME LLIBRE AND CHARA PANTAZI
}

\begin{abstract}
In Geometriae Dedicata 79 (2000), 101-108, Rudolf Winkel conjectured: For a given algebraic curve $f=0$ of degree $m \geqslant 4$ there is in general no polynomial vector field of degree less than $2 m-1$ leaving invariant $f=0$ and having exactly the ovals of $f=0$ as limit cycles. Here we show that this conjecture is not true.
\end{abstract}

\section{INTRODUCTION}

A planar vector field

$$
X=P(x, y) \frac{\partial}{\partial x}+Q(x, y) \frac{\partial}{\partial y}
$$

is polynomial of degree $n$ if $P$ and $Q$ are real polynomials in the variables $x$ and $y$, and the maximum degree of $P$ and $Q$ is $n$.

A periodic orbit of a vector field $X$ in $\mathbb{R}^{2}$ is a limit cycle if it is isolated in the set of all periodic orbits of $X$.

In 1900 Hilbert [4] in the second part of its 16-th problem proposed to find an estimation of the uniform upper bound for the number of limit cycles of all polynomial vector fields of a given degree, and also to study their distribution or configuration in the plane. This has been one of the main problems in the qualitative theory of planar differential equations in the XX century. The contributions of Écalle [2] and Ilyashenko [5] proving that any polynomial vector field has finitely many limit cycles have been the best results in this area. But until now it is not proved the existence of an uniform upper bound. This problem remains open even for the quadratic polynomial vector fields.

If $f=f(x, y)$ is an irreducible polynomial of degree $m$ in the ring $\mathbb{R}[x, y]$, then $f=0$ is an irreducible algebraic curve of degree $m$ in $\mathbb{R}^{2}$.

1991 Mathematics Subject Classification. Primary 14P25, 34C05, 34A34.

Key words and phrases. polynomial vector fields, algebraic limit cycles.

The authors are partially supported by a MCYT grant BFM 2002-04236-C02-02 and by a CIRIT grant number 2001SGR 00173. 
A limit cycle is algebraic of degree $m$ if it is a contained in an irreducible algebraic curve of degree $m$.

Hilbert also asked about the possible distributions of the limit cycles of polynomial vector fields. Recently, it has been proved that any finite configuration of limit cycles is realizable by polynomial vector fields. More precisely, we say that a configuration of limit cycles is a finite set of disjoint simple closed curves of the plane pairwise disjoint. Two configurations of limit cycles are (topologically) equivalent if there is a homeomorphism of $\mathbb{R}^{2}$ applying one configuration into the other. We say that the vector field $X$ realizes a given configuration of limit cycles if the set of all limit cycles of $X$ is equivalent to that configuration. Recently, in [6] it is proved that any configuration of limit cycles is topologically realizable as algebraic limit cycles by a polynomial vector field of a convenient degree.

In [7] Winkel did the following conjecture about the algebraic limit cycles of polynomial vector fields.

Conjecture 1. For a given algebraic curve $f=0$ of degree $m \geqslant 4$ there is in general no polynomial vector field of degree less than $2 m-1$ leaving invariant $f=0$ and having exactly the ovals of $f=0$ as limit cycles.

Here we will work with the one-parameter family of irreducible algebraic curves

$$
f=f(x, y)=\frac{1}{4}+x-x^{2}+p x^{3}+x y+x^{2} y^{2}=0,
$$

of degree $m=4$ with $0<p<1 / 4$. These curves have three connected components, one is an oval and each of the other two is homeomorphic to a straight line, see Figure 1 . We note that the oval of $f=0$ borns at the point $(2,-1 / 4)$ when $p=1 / 4$. Then, when $p$ decreases the oval increases its size and ends having infinite size at the irreducible curve $1 / 4+x-x^{2}+x y+x^{2} y^{2}=0$ when $p=0$.

We must mention that the curve $f=0$ has no singular points, i.e. there is no real solutions of the system $f=0, \partial f / \partial x=0$ and $\partial f / \partial y=$ 0 .

First we will prove that the oval of the curve (2) is the unique limit cycle of a 13-parameter family of polynomial vector fields of degree 5 . Since $2 m-1=7>5$, this provides a counterexample to Conjecture 1 . Many other counterexamples can be constructed changing the algebraic curve $f=0$.

Let $f \in \mathbb{R}[x, y]$. The algebraic curve $f(x, y)=0$ is an invariant algebraic curve of the vector field $X$ if for some polynomial $K \in \mathbb{R}[x, y]$ 


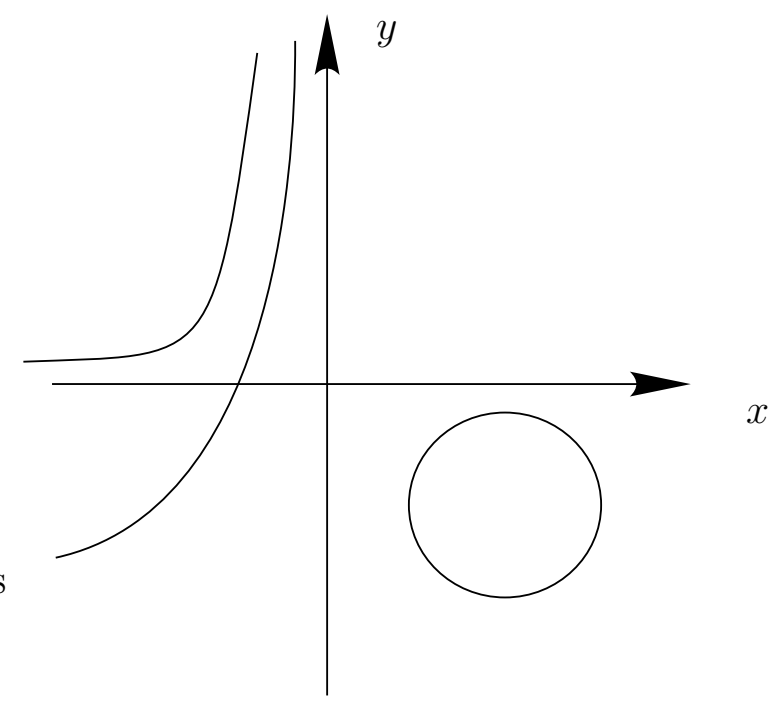

Figure 1. Algebraic limit cycle of degree 4.

we have

$$
X f=P \frac{\partial f}{\partial x}+Q \frac{\partial f}{\partial y}=K f .
$$

The polynomial $K$ is called the cofactor of the invariant algebraic curve $f=0$. We note that since the polynomial system has degree $n$, then any cofactor has at most degree $n-1$. Since on the points of the algebraic curve $f=0$ the gradient $(\partial f / \partial x, \partial f / \partial y)$ of the curve is orthogonal to the vector field $X$, we have that $X$ is tangent to the curve $f=0$. Hence, the curve $f=0$ is formed by trajectories of $X$. This justifies the name of invariant algebraic curve given to the algebraic curve $f=0$ satisfying (3) for some polynomial $K$, because it is invariant under the flow defined by $X$.

Our main result is the following one.

Theorem 2. Let $a, b, c, d$, e and $p$ arbitrary real numbers. Then, the algebraic curve $f=0$ given by (2) is invariant by the 6-parameter family of real polynomial vector fields (1) of degree 5 given by

$$
\begin{aligned}
P & =-2 a f_{2} f_{3} \frac{\partial f_{1}}{\partial y}-2(b+i c) f_{1} f_{3} \frac{\partial f_{2}}{\partial y}-2(b-i c) f_{1} f_{2} \frac{\partial f_{3}}{\partial y} \\
Q & =2 a f_{2} f_{3} \frac{\partial f_{1}}{\partial x}+2(b+i c) f_{1} f_{3} \frac{\partial f_{2}}{\partial x}+2(b-i c) f_{1} f_{2} \frac{\partial f_{3}}{\partial x}
\end{aligned}
$$

where $i=\sqrt{-1}, f_{1}=f, f_{2}=x-d+i(y-e)$ and $f_{3}=x-d-i(y-e)$. Moreover, if ac $\neq 0,0<p<1 / 4$ and the point $(d, e)$ is in the interior 
of the bounded region limited by the oval of $f=0$, then the unique limit cycle of this vector field is the algebraic one formed by the oval of $f=0$.

In Section 2 we present some basic definitions and results that we shall use in Section 3 for proving Theorem 2.

We note that if we do an affine transformation of the polynomial differential system $\dot{x}=P(x, y), \dot{y}=Q(x, y)$, where $P$ and $Q$ are the ones given in the statement of Theorem 2 , and a rescaling of the independent variable, then the polynomial vector fields of degree 5 associated to the new differential systems form a 13-parameter family providing a counterexample to Conjecture 1 .

In fact a weaker counterexample formed by an 8-parameter family of quadratic polynomial vector fields follows from the next theorem proven in [1].

Theorem 3. The quadratic polynomial differential system

$$
\begin{aligned}
& \dot{x}=2\left(1+2 x-2 p x^{2}+6 x y\right), \\
& \dot{y}=8-3 p-14 p x-2 p x y-8 y^{2},
\end{aligned}
$$

with $0<p<1 / 4$ possesses the irreducible invariant algebraic curve $f=0$. Moreover, if $0<p<1 / 4$, then the unique limit cycle of this system is the algebraic one formed by the oval of $f=0$.

\section{Preliminary Definitions}

In this section $U$ will be an open subset of $\mathbb{R}^{2}$. Let $X$ be a $C^{1}$ vector field defined in $U$. A nonconstant $C^{1}$ function $H: U \rightarrow \mathbb{R}$ is a first integral of $X$ in $U$ if $H$ remains constant along the trajectories of $X$ contained in $U$. Then, the level curves $H(x, y)=h$ in $U$ are almost sufficient to describe the phase portrait of $X$ in $U$, since every level curve is the union of orbits of $X$.

A function $R: U \rightarrow \mathbb{R}^{2}$ is an integrating factor for the polynomial vector field $X$ on $U$ if there exists a function

$$
H: U \rightarrow \mathbb{R}
$$

such that

$$
\begin{aligned}
& \dot{x}=R(x, y) P(x, y)=-\frac{\partial H}{\partial y}(x, y), \\
& \dot{y}=R(x, y) Q(x, y)=\frac{\partial H}{\partial x}(x, y) .
\end{aligned}
$$

This is a Hamiltonian system defined in $U$, and the function $H$ is called the Hamiltonian of the system. 
Clearly, from the definition of integrating factor it follows that a function $V: U \rightarrow \mathbb{R}^{2}$ is an inverse integrating factor for the polynomial vector field $X$ on $U$ if there exists a function

$$
H: U \backslash\{V(x, y)=0\} \rightarrow \mathbb{R}
$$

such that

$$
\begin{aligned}
\dot{x} & =\frac{P(x, y)}{V(x, y)}=-\frac{\partial H}{\partial y}(x, y), \\
\dot{y} & =\frac{Q(x, y)}{V(x, y)}=\frac{\partial H}{\partial x}(x, y) .
\end{aligned}
$$

This Hamiltonian system is defined in

$$
U \backslash\{V(x, y)=0\} .
$$

We say that the Hamiltonian $H$ is associated to the inverse integrating factor $V$ and vice versa. Note that given $X$ if we know $H$ we know $V$, and vice versa.

Usually the expression of the inverse integrating factor $V$ is easier than the expression of its associated Hamiltonian $H$. So, for studying the integrability of a planar differential system is better to look for $V$ than to look for $H$. But the main reason for working with the inverse integrating factor is the following result due to Giacomini, Llibre and Viano [3], which will play a main role in our proof of Theorem 2. Here, we provide an easier and direct proof, which also appears in Llibre and Rodríguez [6].

Theorem 4. Let $X$ be a $C^{1}$ vector field defined in the open subset $U$ of $\mathbb{R}^{2}$. Let $V: U \rightarrow \mathbb{R}$ be an inverse integrating factor of $X$. If $\gamma$ is a limit cycle of $X$, then $\gamma$ is contained in $\boldsymbol{\Sigma}=\{(x, y) \in U: V(x, y)=0\}$.

Proof: Due to the existence of the inverse integrating factor $V$ defined in $U$, we have that the vector field $X / V$ is Hamiltonian in $U \backslash \Sigma$. Since the flow of a Hamiltonian vector field preserves the area and in a neighborhood of a limit cycle a flow does not preserve the area, the theorem follows.

\section{Proof of Theorem 2}

A straightforward computation shows that the algebraic curve $f=$ 0 given by (2) is invariant by the polynomial vector field $X$ whose components $P$ and $Q$ are given in the statement of Theorem 2. In fact, 
the cofactor $K$ of $f=0$ is

$$
\begin{aligned}
K= & 4[b e-c d+(c-b d+2 c d-2 b e-c e) x+(b e-b-c d) y+ \\
& (b-2 c-3 c d p+3 b e p) x^{2}+2(b+c) x y-b y^{2}+ \\
& 3 c p x^{3}-(2 b d+2 c e+3 b p) x^{2} y-2(c d-b e) x y^{2}+ \\
& \left.2 b x^{3} y+4 c x^{2} y^{2}-2 b x y^{3}\right] .
\end{aligned}
$$

Now the key point in the proof of Theorem 2 is to show that the unique limit cycle of $X$ is the oval $\gamma$ contained in $f=0$ for $0<p<1 / 4$ when $a c \neq 0$ and $(d, e)$ is a point contained in the interior of the bounded region limited by $\gamma$. In order to prove that, first with another easy computation we check that

$$
V=f \cdot\left[(x-d)^{2}+(y-e)^{2}\right],
$$

and

$$
H=2 a \log f+2 b \log \left[(x-d)^{2}+(y-e)^{2}\right]-4 c \arg [(x-d)+i(y-e)],
$$

are the inverse integrating factor and its associated Hamiltonian for our polynomial vector field $X$.

Since $V$ is polynomial, $V$ is defined in the whole $\mathbb{R}^{2}$. Therefore, by Theorem 4 and since $V(x, y)=0$ if and only if $(x, y) \in\{f=$ $0\} \cup\{(d, e)\}$, it follows that if the vector field $X$ has some limit cycle, this must be the oval $\gamma$ of $f=0$. Now, we shall prove that this oval is a limit cycle. Hence, Theorem 2 will be proved.

We observe that $P$ and $Q$ are real because they can be written as

$$
\begin{aligned}
P= & (b e-c d)+\left[c+4(b e-c d)-2 a\left(d^{2}+e^{2}\right)\right] x-b y+ \\
& 4[c+(a+c) d-b e] x^{2}-4[b+c d-(a+b) e] x y- \\
& 2[a+2 c+2 p(c d-b e)] x^{3}+4\left[b+c-a\left(d^{2}+e^{2}\right)\right] x^{2} y- \\
& 2(a+2 b) x y^{2}+4 c p x^{4}+4(2 a d-b p) x^{3} y- \\
& 4(c d-2 a e-b e) x^{2} y^{2}-4 a x^{4} y+4 c x^{3} y^{2}-4(a+b) x^{2} y^{3}, \\
Q= & 2 a\left(d^{2}+e^{2}\right)-b d-c e+[b-4((a+b+a d) d+(c+a e) e)] x+ \\
& {\left[c+2 a\left(d^{2}-2 e+e^{2}\right)\right] y-4(-c+a d+b d-2 a e+c e) x y+} \\
& 2\left[a+2 b+2 d(2 a+b)+2 c e+3 a p\left(d^{2}+e^{2}\right)\right] x^{2}+2 a(1-2 e) y^{2}- \\
& 4(a+b+3 a d p+b d p+c e p) x^{3}+2(a+2 b-2 c-6 a e p) x^{2} y+ \\
& 4\left(-a+c+a d^{2}+a e^{2}\right) x y^{2}+2 a y^{3}+ \\
& 2(3 a+2 b) p x^{4}+4 c p x^{3} y-2(4 a d+2 b d+2 c e-3 a p) x^{2} y^{2}- \\
& 8 a e x y^{3}+4(a+b) x^{3} y^{2}+4 c x^{2} y^{3}+4 a x y^{4} .
\end{aligned}
$$


Since $f=0$ is an invariant algebraic curve of the vector field $X$, the oval $\gamma$ is formed by solutions of $X$. Now we shall prove that on the oval $\gamma$ there are no singular points of $X$ and, therefore, $\gamma$ will be a periodic orbit. Assume that $\left(x_{0}, y_{0}\right)$ is a singular point of $X$ contained on the oval $\gamma$; i.e., $P\left(x_{0}, y_{0}\right)=Q\left(x_{0}, y_{0}\right)=f\left(x_{0}, y_{0}\right)=0$. From (4) we have that

$$
\begin{aligned}
& P\left(x_{0}, y_{0}\right)=-2 a f_{2}\left(x_{0}, y_{0}\right) f_{3}\left(x_{0}, y_{0}\right) \frac{\partial f}{\partial y}\left(x_{0}, y_{0}\right)=0 \\
& Q\left(x_{0}, y_{0}\right)=2 a f_{2}\left(x_{0}, y_{0}\right) f_{3}\left(x_{0}, y_{0}\right) \frac{\partial f}{\partial x}\left(x_{0}, y_{0}\right)=0 .
\end{aligned}
$$

Since $a \neq 0$ and $f_{2}\left(x_{0}, y_{0}\right) f_{3}\left(x_{0}, y_{0}\right)=\left(x_{0}-d\right)^{2}+\left(y_{0}-e\right)^{2} \neq 0$, we obtain that $\frac{\partial f}{\partial x}\left(x_{0}, y_{0}\right)=0$ and $\frac{\partial f}{\partial y}\left(x_{0}, y_{0}\right)=0$. This is not possible, otherwise the point $\left(x_{0}, y_{0}\right)$ would be a singular point of the algebraic curve $f=0$, and this curve has no singular points when $0<p<1 / 4$. Hence, the oval $\gamma$ is a periodic orbit of the vector field $X$. Now, we shall prove that $\gamma$ will be a limit cycle, and this will complete the proof of Theorem 2.

We define the first integral $\bar{H}$ of $X$ as follows

$$
\bar{H}=e^{H}=f^{2 a}\left[(x-d)^{2}+(y-e)^{2}\right]^{2 b} e^{-4 c \arg [(x-d)+i(y-e)]} .
$$

Then we note that the oval $\gamma$ and the point $(d, e)$ are in the level $\bar{H}(x, y)=0$, and that they are the unique orbits of $X$ in this level. Now suppose that $\gamma$ is not a limit cycle. Then, there is a periodic orbit $\gamma^{\prime}=\{(x(t), y(t)): t \in \mathbb{R}\}$ different from $\gamma$ and sufficiently close to $\gamma$ such that the bounded component $B$ limited by $\gamma^{\prime}$ contains the point $(d, e)$.

As $\gamma^{\prime}$ is different from $\gamma$, there exists $h \neq 0$ such that

$$
\bar{H}(x(t), y(t))=f^{2 a}(x(t), y(t))\left[(x(t)-d)^{2}+(y(t)-e)^{2}\right]^{2 b} e^{-4 c \theta(t)}=h,
$$

where $\theta(t)=\arg [(x(t)-d)+i(y(t)-e)]$. The function $f^{2 a}(x(t), y(t))[(x(t)-$ $\left.d)^{2}+(y(t)-e)^{2}\right]^{2 b}$ is bounded on $\gamma^{\prime}$. Clearly, since the point $(d, e)$ is in the bounded region limited by $\gamma^{\prime}$ the angle $\theta(t)$ tends to either $+\infty$ or $-\infty$, when $t \rightarrow+\infty$. Since $c \neq 0$, this fact is in contradiction with equality $\bar{H}(x(t), y(t))=h \neq 0$. Consequently, we have proved that $\gamma$ is a limit cycle. In short, Theorem 2 is proved.

\section{REFERENCES}

[1] J. Chavarriga, H. Giacomini and J. Llibre, Uniqueness of algebraic limit cycles for quadratic systems, J. Math. Anal. Appl. 261 (2001), 85-99.

[2] J. ÉCALLE, Introduction aux fonctions analysables et preuve constructive de la conjecture de Dulac, Hermann, 1992. 
[3] H. Giacomini, J. Llibre and M. Viano, On the nonexistence, existence and uniqueness of limit cycles, Nonlinearity 9 (1996), 501-516.

[4] D. Hilbert, Mathematische Probleme, Lecture, Second Internat. Congr. Math. (Paris, 1900), Nachr. Ges. Wiss. G"ttingen Math. Phys. KL. (1900), 253-297; English transl., Bull. Amer. Math. Soc. 8 (1902), 437-479.

[5] Yu. Ilyashenko, Finiteness theorems for limit cycles, Translations of Math. Monographs 94, Amer. Math. Soc., 1991.

[6] J. Llibre and G. RodríGuez, Configurations of limit cycles and planar polynomial vector fields, to appear in J. of Differential Equations.

[7] R. Winkel, A transfer principle in the real plane from nonsingular algebraic curves to polynomial vector fields, Geometriae Dedicata 79 (2000), 101-108.

* Departament de Matemàtiques, Universitat Autònoma de Barcelona, 08193 Bellaterra, Barcelona, Spain

E-mail address: jllibre@mat.uab.es, hpant@mat.uab.es 